\title{
Short-term Heart Rate Variability Analysis in Healthy Dogs of Different Ages
}

\author{
Luciene Martinello ${ }^{1}$, Amanda Sarita Cruz Aleixo ${ }^{1}$, Felipe Gazza Romão ${ }^{1}$, Mayra de Castro Ferreira Lima', \\ Miriam Tsunemi ${ }_{\odot}^{2}$, Simone Biagio Chiacchio ${ }_{\odot}^{1}$, Moacir Fernandes de Godoy ${ }^{3}$ \& Maria Lucia Gomes Lourenço ${ }^{1}$
}

\begin{abstract}
Background: The modulation of heart rate by autonomic nervous system may be evaluated by the heart rate variability (HRV), which illustrates the fluctuations between RR intervals. To evaluate this analysis, the intervals between 2 QRS complexes are measured. In general, high HRV values are expected in healthy individuals; otherwise, low values are indicative of organism dysfunction. Studies conducted in healthy humans show that HRV suffers reduction with ageing and that there is autonomic immaturity in neonates. The aim of this study was to describe the characteristic pattern of cardiac autonomic behavior in healthy dogs in different age groups through short-term HRV analysis.

Materials, Methods \& Results: A total of 87 healthy dogs were studied. HRV was analyzed in time and frequency domain, using Holter and heart rate monitor. It was observed that puppies (below one year old) presented a lower parasympathetic predominance and, consequently, lower HRV values on time domain (SDNN, PNN50\% e RMSSD) compared to the other 2 groups and on frequency domain (LF, HF and LF/HF) compared to the adult animals group (between 1 and 7-year-old), which presented higher HRV values when compared to the other groups. Elderly dogs (over 8-year-old) exhibited a natural tendency to decrease cardiac parasympathetic HRV indexes.

Discussion: The use of the HRV method as a prognostic index and as an arrhythmogenic marker for various canine heart diseases presents interesting perspectives. However, before it may be employed for these purposes, a better understanding should be established regarding the physiological behavior of autonomic cardiac modulation in different age groups to serve as a basis for future analyses. This study observed that puppies presented higher values for HR and, therefore, shorter RR intervals than the other groups (adult and elderly dogs), what was observed on Holter and heart rate monitor methods (HRM). There were significant differences between puppies and the other 2 groups (adults and elderly) for all time-domain variables using both methods (Holter and HRM methods). SDNN was significantly lower in puppies compared to adults and elderly dogs. In addition, both RMSSD and PNN50\%, which were more reliable over shorter periods of time, also presented means and medians that were significantly lower in puppies. Regarding frequency-domain HRV parameters observed on Holter method, these indexes were decreased on the elderly group compared to adult dogs, which is a possible effect of aging. Also, puppies revealed lower frequency-domain HRV parameters on both methods when compared to adult dogs. The influence of age on HRV is possibly related to the stage of development of an individual, starting at conception up to the maturity in relation to the mechanisms that cause variations in HR. There are studies in humans that suggest a gradual increase in parasympathetic activity during childhood, followed by a steady decrease as aging occur. The present study observed the same pattern in dogs. The balance between sympathetic and parasympathetic systems is influenced by age in dogs, which alters HRV values in the short-term. The HRV method's analysis is relatively simple and non-invasive for assessing cardiac autonomic function; also, it is widely used in human medicine as a risk measure for sudden cardiac death. The 24-hour HRV analysis is highly challenging, as it is time-consuming, expensive, delays diagnosis, and has a large number of artifacts; in this way, standards for its short-term analysis were developed.
\end{abstract}

Keywords: cardiology, autonomic nervous system, heart rate monitor, Holter.

DOI: $10.22456 / 1679-9216.118578$

Accepted: 14 December 2021

Published: 4 January 2022 CORRESPONDENCE: L. Martinello [lucienemartinello@ hotmail.com]. São Paulo State University (Unesp). Rubião Júnior District. CEP 18618-970 Botucatu, SP, Brazil. 


\section{INTRODUCTION}

The primary function of the ANS (autonomic nervous system) is to maintain homeostasis in internal environment of the body, unconsciously regulating organic functions such as blood pressure and heart rate (HR), and it is divided into 2 large subsystems, sympathetic nervous system (SNS) and parasympathetic nervous system (PNS) [17].

Due to the influence of the ANS on the sinus node, there are oscillations between heart beats (RR intervals), which is called HRV (heart rate variability) $[2,20]$. Low HRV is usually an indicator of abnormal and insufficient ANS adaptation, which indicates physiological dysfunction. Conversely, high HRV is an indicator of good adaptation, characterizing a healthy individual with sufficient autonomic control. However, there are some clinical effects that increase HRV considerably, such as cardiac arrythmias, a phenomenon that cannot be considered normal [1,4]. HRV decreases with aging in healthy humans, reflecting a loss of efficiency in autonomic adjustments $[8,16]$.

The use of the HRV method as a prognostic index and as an arrhythmogenic marker for various canine heart diseases presents interesting perspectives. However, before it may be employed for these purposes, a better understanding should be established regarding the physiological behavior of autonomic cardiac modulation in different age groups to serve as a basis for future analyses. Therefore, this study aims to describe the characteristic patterns of the short-term autonomic cardiac behavior in healthy dogs belonging to different age groups, employing 2 different devices to obtain the HRV data: a Holter monitor device and a HR monitor.

\section{MATERIALS AND METHODS}

\section{Study design and animals}

The owners signed free and informed consent terms. A total of $87 \mathrm{dogs}$ [38 males and 49 females], without breed distinction, were included in the study. Estimation of the sample size (n) was calculated at $80 \%$ statistical power. The animals were divided into 3 distinct groups according to their age:

- Group 1 (G1): 30 healthy dogs younger than 1-year-old.

- Group 2 (G2): 30 healthy dogs between 1 and 7-year-old.
- Group 3 (G3): 27 healthy dogs older than 8-year-old.

The dogs included in the study were previously examined, and those presenting echocardiographic and/or electrocardiographic alterations resulting in hemodynamic abnormalities were excluded from the study. Additionally, brachycephalic dogs, animals with previous cardiac disease, and dogs with cardiac remodeling were excluded. Animals with arrhythmias or conduction disorders in a conventional ECG or from a Holter monitor were also excluded.

The exams were conducted during the day, and all animals underwent a previous physical examination followed by a specific evaluation of the cardiovascular system. An echocardiographic examination was then conducted, and animals meeting the study criteria were selected and divided into groups according to their age groups, as described above.

Exams

The animals were initially physically restrained and kept in right lateral recumbency and, later, in left lateral recumbency to perform an echocardiogram. They were then released for $10 \mathrm{~min}$ and again physically restrained for electrocardiographic examination together with measurement with the HR monitor for $20 \mathrm{~min}$.

The analysis with the HR monitor was conducted concurrently with the electrocardiogram to enable the visualization of possible arrhythmias and conduction disorders, in which case the animals were excluded from the study.

The measurement of the RR interval was conducted using a Polar ${ }^{\circledR 1}$ HR monitor, a validated method for dogs [6,13], consisting of an elastic band with 2 areas of increased electric sensitivity and a small central device responsible for recording the heart beats. The data obtained were transmitted via radio waves to a Polar ${ }^{\circledR}$ wristwatch and then to a computer via USB. As the device was designed to be used in adult humans, a small adaptation is described in the literature for its use in human neonates [18], and this adaptation was maintained for dogs in the present study due to the lower size of the canine thorax compared with an adult human thorax. For this purpose, the area was shaved to ensure better contact with the electrodes and reduce interference. Two electrodes coupled to the Polar ${ }^{\circledR}$ HR monitor1 were then placed one on each side in the sternal region, at the level of the fourth intercostal 
space, making it unnecessary to use elastic bands and allowing the HR monitor to be attached to the thorax of the animal.

The RR intervals acquired by the HR monitor were transferred to a computer, visualized with Polar FlowSync ${ }^{\circledR 1}$ and corrected manually. After correction, the RR intervals were transferred to Kubios ${ }^{\circledR 2}$, and then the "smooyhn priors" filter was applied.

After HR monitor removal, a Holter monitor was placed on the animals for $90 \mathrm{~min}$. During monitoring, the owners were advised to let the animal perform its normal routine as usual at home. The Holter monitor used was a Cardiolight ${ }^{\circledR 3}$ digital device for humans, which records via 3 channels $(\mathrm{C} 1, \mathrm{C} 2$, and $\mathrm{C} 3)$, storing the information on an SD Card. The data collected were analyzed with the appropriate software (Software CardioSmart $\left.550^{\circledR}\right)^{3}$ for the device, which visualized and interpreted RR intervals. Since the device was designed to be used on humans, all events were verified individually to ensure a high precision of the results. Time and frequency-domain indexes were obtained.

After analysis of data from Holter monitors and HR monitors, the mean HR, the mean RR interval during the recording, and the HRV data were transferred. These intervals were digitally filtered to eliminate premature beats and then manually reanalyzed to delete residual artifacts. Only time series with less than $10 \%$ artifacts were included for analysis.
The HRV was calculated in the time and frequency domains. For the time domain, the following indexes were registered: SDNN (standard deviation of all normal RR intervals, expressed in milliseconds), pNN50\% (proportion of differences exceeding $50 \mathrm{~ms}$ [milliseconds] between adjacent normal RR intervals and RMSSD (root mean square of successive differences).

For the frequency domain, a spectral analysis was conducted through the Fast Fourier Transform (FFT) algorithm, and the following indexes were registered: high frequency (HF) varying from 0.15 to $0.4 \mathrm{~Hz}$ (Hertz); low frequency (LF) varying from 0.04 to 0.15 $\mathrm{Hz}$; and very low frequency (VLF) as defined by other authors for dogs $[8,16]$. All variables are expressed in normalized units (n.u.).

\section{Statistical analysis}

The data obtained were then analyzed using statistical tests. Variable (medians) comparison between age groups was conducted using the KruskalWallis test (for nonparametric data) since the normality assumptions (Shapiro-Wilks test) were not met. For cases with statistically significant differences, multiple comparisons were drawn between age groups. For all statistical analysis, a $P<0.05$ was considered statistically significant, according to Dunn's test corrected by Bonferroni method (Tables 1 and 2).

Table 1. HRV parameters obtained through the Heart Rate Monitor method in healthy dogs, analyzed in Kubios ${ }^{\circledR}$ software and organized according to age groups.

\begin{tabular}{cccc}
\hline & $\begin{array}{c}\text { Group 1 } \\
<1 \text { year }(\mathrm{n}=30)\end{array}$ & $\begin{array}{c}\text { Group 2 } \\
1.1-7.9 \text { years }(\mathrm{n}=30)\end{array}$ & $\begin{array}{c}\text { Group 3 } \\
>8 \text { years }(\mathrm{n}=27)\end{array}$ \\
\hline Parameter & Mean \pm CI (SD) median & Mean \pm CI (SD) median & Mean \pm CI (SD) median \\
\hline HR (bpm) & $164.30 \pm 11.67(32.61) 170.00^{\mathrm{a}}$ & $109.00 \pm 8.07(22.55) 109.50^{\mathrm{b}}$ & $123.48 \pm 9.95(26.37) 118.00^{\mathrm{b}}$ \\
Mean RR & $381.93 \pm 31.41(87.78) 353.00^{\mathrm{a}}$ & $572.30 \pm 39.73(111.03) 547.50^{\mathrm{b}}$ & $508.44 \pm 42.69(113.17) 510.00^{\mathrm{b}}$ \\
SDNN (msec) & $37.80 \pm 7.96(22.24) 32.10^{\mathrm{a}}$ & $73.47 \pm 11.53(32.30) 69.40^{\mathrm{b}}$ & $85.66 \pm 15.81(41.92) 83.00^{\mathrm{b}}$ \\
RMSSD (msec) & $45.99 \pm 11.23(31.39) 34.00^{\mathrm{a}}$ & $103.30 \pm 19.23(53.73) 99.50^{\mathrm{b}}$ & $115.05 \pm 25.27(66.99) 109.50^{\mathrm{b}}$ \\
PNN50\% & $18.84 \pm 6.49(18.13) 13.29^{\mathrm{a}}$ & $55.17 \pm 6.01(16.79) 57.41^{\mathrm{b}}$ & $50.40 \pm 9.08(24.06) 54.51^{\mathrm{b}}$ \\
LF (n.u.) & $47.04 \pm 5.19(14.49) 45.35^{\mathrm{a}}$ & $33.47 \pm 4.33(12.10) 32.63^{\mathrm{b}}$ & $37.85 \pm 5.36(14.20) 36.72^{\mathrm{b}}$ \\
HF (n.u.) & $52.56 \pm 5.14(14.37) 53.84^{\mathrm{a}}$ & $65.66 \pm 4.27(11.92) 66.35^{\mathrm{b}}$ & $61.67 \pm 5.25(13.92) 63.13^{\mathrm{b}}$ \\
LH/HF & $1.12 \pm 0.35(0.98) 0.84^{\mathrm{a}}$ & $0.57 \pm 0.16(0.44) 57.41^{\mathrm{b}}$ & $0.71 \pm 0.20(0.52) 0.58^{\mathrm{b}}$
\end{tabular}

Confidence Interval $(\mathrm{CI})=$ mean \pm (standard deviation* 1.96). Matching letters mean that the medians did not present significant difference while different letters mean that the medians presented statistically significant differences. Differences between age groups were significant when $P<0.05$. Test employed: Kruskal-Wallis. bpm: beats per minute; HF: high frequency; HR: heart rate; LF: low frequency; n.u.: normalized units; PNN50: proportion of differences exceeding $50 \mathrm{msec}$ between adjacent normal RR intervals; RMSSD: root mean square of successive differences; RR: interval between two consecutive R waves or average RR of the entire record; SD: standard deviation; SDNN: standard deviation of all normal RR intervals. 
Table 2. HRV parameters obtained through the holter method in healthy dogs organized according to age groups.

\begin{tabular}{cccc}
\hline & Group 1 & Group 2 & Group 3 \\
& $<$ year $(\mathrm{n}=30)$ & $1.1-7.9$ years $(\mathrm{n}=30)$ & $>8$ years $(\mathrm{n}=27)$ \\
\hline Parameter & Mean \pm CI (SD) median & Mean \pm CI (SD) median & Mean \pm CI (SD) median \\
\hline HR (bpm) & $151.73 \pm 11.54(32.25) 157.00^{\mathrm{a}}$ & $96.70 \pm 5.36(14.98) 95.52^{\mathrm{b}}$ & $111.77 \pm 11.83(31.36) 110.00^{\mathrm{b}}$ \\
Mean NN & $449.83 \pm 38.67(108.05) 420.50^{\mathrm{a}}$ & $673.06 \pm 37.16(103.85) 675.50^{\mathrm{b}}$ & $620.22 \pm 67.29(178.39) 578.00^{\mathrm{b}}$ \\
SDNN (msec) & $78.03 \pm 16.92(47.28) 75.50^{\mathrm{a}}$ & $203.86 \pm 27.47(78.09) 185.00^{\mathrm{b}}$ & $167.02 \pm 35.05(92.93) 137.00^{\mathrm{b}}$ \\
RMSSD (msec) & $73.93 \pm 18.53(51.76) 65.00^{\mathrm{a}}$ & $203.20 \pm 33.16(92.65) 192.00^{\mathrm{b}}$ & $170.66 \pm 49.83(132.11) 110.00^{\mathrm{b}}$ \\
PNN50\% & $24.15 \pm 6.57(18.35) 55.62^{\mathrm{a}}$ & $61.09 \pm 5.52(15.43) 64.80^{\mathrm{b}}$ & $43.43 \pm 9.67(25.64) 40.01^{\mathrm{c}}$ \\
LF (nu) & $55.13 \pm 4.46(12.45) 55.09^{\mathrm{a}}$ & $32.81 \pm 5.55(15.51) 32.52^{\mathrm{b}}$ & $46.58 \pm 7.70(20.40) 47.2^{\mathrm{a}}$ \\
HF (nu) & $50.03 \pm 7.38(20.62) 46.28^{\mathrm{a}}$ & $66.00 \pm 5.66(15.81) 32.52^{\mathrm{b}}$ & $53.49 \pm 7.74(20.53) 52.75^{\mathrm{a}}$ \\
LH/HF & $1.43 \pm 0.34(0.95) 1.32^{\mathrm{a}}$ & $0.63 \pm 0.17(0.46) 0.59^{\mathrm{b}}$ & $1.26 \pm 0.40(1.05) 0.93^{\mathrm{a}}$ \\
\hline
\end{tabular}

Confidence Interval $(\mathrm{CI})=$ mean \pm (standard deviation* 1.96). Matching letters mean that the medians did not present significant difference while different letters mean that the medians presented statistically significant differences. Differences between age groups were significant when $P<0.05$. Test employed: Kruskal-Wallis. bpm: beats per minute; HF: high frequency; HR: heart rate; LF: low frequency; n.u.: normalized units; PNN50: proportion of differences exceeding $50 \mathrm{msec}$ between adjacent normal RR intervals; RMSSD: root mean square of successive differences; RR: interval between two consecutive $\mathrm{R}$ waves or average RR of the entire record; SD: standard deviation; SDNN: standard deviation of all normal RR intervals.

\section{RESULTS}

The trial was a randomized study, with age being the only grouping criterion. A total of 91 dogs were included in the study, but 4 had to be excluded because they could not be restrained for the examinations.

There were significant differences (Tables 1 and 2) between G1 and the other 2 groups for HR, $\mathrm{RR}$ interval, and all time-domain variables using both methods. Animals in G1 (puppies) presented a higher mean HR and, therefore, shorter RR intervals. SDNN was significantly lower in G1 than in G2 and G3. Additionally, both RMSSD and PNN50\%, which were more reliable over shorter periods of time, also presented means and medians that were significantly lower in G1.

The mean and median values for the variables SDNN and RMSSD did not vary significantly between G2 and G3 for both methods (Tables 1 and 2), but the variable PNN50\% presented a significant difference between the groups using the Holter method (Table 2). With the HR monitor method, G3 presented higher values than $\mathrm{G} 2$ for all variables in the time domain, except for PNN50\%, and animals from group G2 were more stressed during physical restraint than animals from G3. With the Holter method, G3 presented lower values than $\mathrm{G} 2$ for all parameters in the time domain. The frequency-domain HRV indexes, expressed in normalized units (n.u.), did not present significant differences between G2 and G3 with the HR monitor method for any variables (Table 1), but the Holter method presented significant differences for these parameters, with $\mathrm{G} 3$ presented higher LF values, lower HF values, and a lower LF/HF ratio than G2 (Table 2 ). In the comparison between $\mathrm{G} 1$ and G3, there was a significant difference with the HR monitor but not with the Holter method (Tables 1 and 2).

In the comparison between G1 and G3, there were significant differences in all variables for these indexes, which was not observed using the Holter method (Tables 1 and 2). In all groups, HF values were higher than LF values, except in G1 (puppies), which presented lower HF values than LF values, a result that was observed for HRV obtained using the Holter method. G1 also presented higher for LF values and LF/ HF ratios, but lower HF values were found compared with the other groups.

The results of the HRV analyses and the comparison of the time-domain and frequency-domain variables (n.u.) across the groups for the HR monitor method are presented in Table 1, while the results for the Holter method are presented in Table 2. The proposed reference values for the analyzed parameters are expressed as the mean $\pm 95 \%$ confidence intervals (CI), with the standard deviations and medians for each variable.

\section{DISCUSSION}

This study observed that 30 puppies (G1) presented higher values for HR and, therefore, shorter RR intervals than the other groups using both methods, supporting the findings described by other authors 
[7,15,19], who observed mean HRs in puppies exceeding $160 \mathrm{bpm}$. Another study analyzed the HR of 10,849 healthy dogs and observed that neonates presented a lower vagal tone than adult dogs, probably due a higher sensitivity to norepinephrine in these dogs [12]. Another study [9] also observed that 30 dogs younger than 1 year-old presented higher HRs than older dogs, which helps support the notion that puppies have physiologically higher HRs.

A study assessing the time-domain HRV in 48 adult dogs with varying body weights observed lower SDNN and RMSSD values in the group of animals younger than 5 years old, although without a significant difference [5]. One possible limiting factor for the research could have been the broad age groups, and as such, this study attempted to define narrower age groups. In the present study, puppies (G1) presented lower values for time-domain HRV indexes than the other groups. Thus, the animals in G1 presented fewer fluctuations in HR in comparison to the median for all time-domain variables and, therefore, lower HR variability, which could be explained by puppies being more excitable than older dogs.

ANS activity has been the focus of recent studies in several animal species (canines, birds, pigs, equines, and humans), and it is used to investigate emotional states, which have an impact on HRV since the indexes reflect a reduced parasympathetic tonus associated with higher sympathetic stimuli due to stress [21]. Animals in G2 presented lower values for SDNN and RMSSD in the HR monitor method than animals in G3 due to the increased sympathetic tonus, although no statistical difference has been observed.

Animals in G3, when evaluated by the Holter method (without physical restraint and at home), presented lower time-domain indexes than G2, which is in line with another study [3] showing a decrease in these indexes in older animals, although without a significant difference. A study conducted with humans revealed a natural tendency towards a progressive reduction in the parasympathetic cardiac markers for $\mathrm{HRV}$, which is probably related to the aging process [8]. It is important to note that results could be different with a different HR monitor that would allow recording without contention.

The frequency-domain variables obtained through the Holter method revealed that elderly dogs presented, during their daily routine, lower HRV than younger dogs. A study [3] with the Holter method observed a reduced HF index in older dogs, although without significant difference, which differs from the results observed in this study. However, there were no statistically significant differences with the HR monitor method between G2 and G3, although the LF index, which reflects sympathetic activity, was higher in G3. Based on results, this possible effect of aging over the frequency-domain HRV parameters appears to be confirmed.

The autonomic and humoral regulation of HR in response to different stimuli is illustrated by HRV. Autonomic control of the hearts of healthy dogs is predominantly influenced by parasympathetic tone, which results in higher HRV [3]. The puppies presented considerably higher values for LF than the other groups, with a much more marked difference shown by the Holter method. This phenomenon may be explained by the varying $\mathrm{HF}$ values according to the respiratory rate. Since the Holter examination was conducted during the animals' daily routines, the respiratory rate was lower than during the HR monitor test, when the animals were physically restrained. This result shows that sympathetic activity is predominant in puppies.

Premature neonates present a less complex HRV behavior, both in the time and frequency domains [18], which may be interpreted as an indication of lower neurological development at this point in their life. Another study in humans reported an increase in sympathetic activity (LF) in individuals under 2 years-old, which could be explained by the tendency for a much higher LF than HF band variable in children [10]. HRV has been assessed in animals under 2 years-old [3], but no differences were observed in this group compared with other animals, probably due to their slightly older age ( 1 to 2 years-old). The present study revealed higher sympathetic activity in healthy animals under 1-year-old.

The influence of age on HRV is possibly related to the stage of development of an individual, starting at conception up to the maturity in relation to the mechanisms that cause variations in HR. There are studies in humans that suggest a gradual increase in parasympathetic activity during childhood, followed by a steady decrease as aging occurs [10]. The present study observed the same pattern in dogs.

Another study assessed 354 healthy people at ages between 3-month and 89-year-old, observing 
peak values for LF and HF in young adults [14]. Our study observed peak values in G2 (young adults), with higher HF values (parasympathetic), lower LF values (sympathetic), and a lower LF/HF ratio than in the other groups, revealing higher HRV with a parasympathetic predominance in this group for both methods.

A study in humans assessed HRV across different age groups and observed that the group presenting the autonomic behavior more in line with homeostasis comprised healthy young adults who presented higher HRV values, which in turn decreased with age. The SDNN variable has been extensively studied in humans and usually presents lower values in elderly and sick individuals. The same study noted that individuals progress towards the onset of diseases as SDNN decreases [11]. In our research using the Holter method, older animals also presented a natural tendency towards reduced SDNN values, indicating that these might also decrease before the onset of diseases in dogs. No similar studies were found with dogs, and this remains an unexplored field in veterinary medicine.

The limitations faced in this study include the difficulty in obtaining the data with the HR monitor, since it requires the animals to be physically restrained to avoid interference. As such, some animals were excluded from the study because it was not possible to restrain them. Another limitation was the inability to adjust the frequency bands $(\mathrm{Hz})$ for the canines using the Holter software, which does not allow such changes. One of the initial goals of the study was to precisely compare the Holter and HR monitors, which was not done because of these limitations.

This line of research is still novel and thoughtprovoking in veterinary medicine, with further studies needed to assess its applicability in dogs. There is also a need for specific equipment to be developed for this species, since both methods have been shown to be difficult when measuring HRV in dogs. Therefore, equipment that suffers less interference from movement, with software that allows an adjustment for the values for different species, should be developed for use in veterinary medicine. This study aimed at describing the time- and frequency-domain HRV in healthy animals, since establishing patterns for healthy individuals of different age groups would facilitate our understanding regarding the autonomic imbalance caused by different clinical morbidities.

\section{CONCLUSION}

Short-term HRV showed an influence of age on its parameters, with greater sympathetic predominance in puppies, greater parasympathetic predominance in young animals, and a physiological decrease in HRV associated with aging. Elderly dogs and puppies presented lower HRV index values, which could be mistaken for indicating diseased animals; however, these alterations were physiological, thus making it extremely important to know the HRV patterns at different ages for future analyses under clinical conditions. Both methods (using a Holter or HR monitor) allowed the short-term analysis of HRV in dogs.

\section{MANUFACTURERS}

${ }^{1}$ Polar Electro Oy. Kempele, Finland.

${ }^{2}$ Kubios. Kuopio, Finland.

${ }^{3}$ Cardios Equipamentos Avançados em Holter e MAPA. São Paulo, SP, Brazil.

Ethical approval. Ethics Commission on Animal Use at the School of Veterinary Medicine and Animal Sciences at UNESP - Botucatu, São Paulo, Brazil (protocol number 204/2017).

Declaration of interest. The authors report no conflicts of interest. The authors alone are responsible for the content and writing of the paper.

\section{REFERENCES}

1 Abreu L.C. 2012. Heart rate variability as a functional marker of development. Journal of Human Growth and Development. 22(3): 279-282.

2 Bobkowski W., Stefaniak M.E., Krauze T., Gendera K., Wykretowicz A., Piskorski J. \& Guzik P. 2017. Measures of Heart Rate Variability in 24h ECGs Depend on Age but Not Gender of Healthy Children. Frontiers in Physiology. 8: $1-14$.

3 Bogucki S. \& Noszczyk-Nowak A. 2015. Short-term heart rate variability (HRV) in healthy dogs. Polish Journal of Veterinary Sciences.18(2): 307-312.

4 Bogucki S. \& Noszczyk-Nowak A. 2017. Short-term heart rate variability in dogs with sick sinus syndrome or chronic mitral valve disease as compared to healthy controls. Polish Journal of Veterinary Sciences. 20(1): 167-172. 
5 Cruz-Aleixo A.S., Alfonso A., Oba E., Souza F.F., Cruz R.K.S., Fillippi M.G., Chiacchio S.B., Tsunemi M. \& Lourenço M.L.G. 2017. Scaling Relationships Among Heart Rate, Electrocardiography Parameters, and Body Weight. Topics in Companion Animal Medicine. 32(2): 66-71.

6 Essner A., Sjostrom R., Ahlgrn E. \& Lindmark B. 2013. Validity and Reability of Polar RS800CX Hear Rate Monitor, Measuring Heart Rate in dogs During Standing Position and at Trot on a Treadmill. Physiology \& Behavior. 10: 114-115.

7 Faria E.G., Nogueira S.S.S. \& Sousa M.G. 2009. Assessment of non-spectral heart rate variability in newborn dogs and cats. Medvep - Revista Científica de Medicina Veterinária. 7(22): 354-356.

8 Fechine B.R.A. \& Trompieri N. 2012. O processo de envelhecimento: as principais alterações que acontecem com o idoso com o passar dos anos. International Scientific Journal. 1(7): 106-132.

9 Ferasin L., Amodo A. \& Murray J.L. 2010. Lack of correlation between canine heart rate and body size in veterinary clinical practice. Journal of Small Animal Practice. 51: 412-418.

10 Finley J.P. \& Nugent S.T. 1995. Heart rate variability in infants, children and young adults. Journal of the Autonomic Nervous System. 54: 103-108.

11 Godoy M.F. \& Gregório M.L. 2019. Diagnostic Relevance of Recurrence Plots for the Characterization of Health, Disease or Death in Humans. Journal of Human Growth and Development. 29(1): 39-47.

12 Hezzell M.J., Humm K., Dennis S.G., Agee L. \& Boswood A. 2013. Relationships between heart rate and age, body weight and breed in dogs. Journal of Small Animal Practice. 54: 318-324.

13 Jonckheer-Sheehy V.S.M., Vinke C.M. \& Ortolani A. 2012. Validation of a Polar human heart rate monitor for measuring heart rate and heart rate variability in adult dogs under stationary conditions. Journal of Veterinary Behavior. 7 : 205-212.

14 Korkushko O.V., Shatilo V.B., Plachinda Y.U.I. \& Shatilo T.V. 1991. Autonomic control of cardiac chronotropic function in man as a function of age: assessment by power spectral analysis of heart rate variability. Journal of Autonomic Nervous System. 32: 191-198.

15 Nogueira S.S.S., Farla E.G. \& Souza M.G. 2010. Avaliação do eletrocardiograma em cães e gatos neonatos. Revista Científica de Medicina Veterinária. 8(24): 101-107.

16 Paschoal M.A., Volanti V.M., Pires C.S. \& Fernandes F.C. 2006. Variabilidade da frequência cardíaca em diferentes faixas etárias. Brazilian Journal of Physical Therapy. 10(4): 413-419.

17 Sá J.C.F., Costa E.C., Silva E. \& Azevedo G.D. 2013. Variabilidade da frequência cardíaca como método de avaliação do sistema nervoso autônomo na síndrome dos ovários policísticos. Revista Brasileira de Ginecologia e Obstetrícia. 35(9): 421-426.

18 Selig F.A., Tonolli E.R., Silva E.V.C.M. \& Godoy M.F. 2011. Variabilidad de la frecuencia cardíaca en neonatos prematuros y de término. Aquivos Brasileiros de Cardiología. 96(6): 443-449.

19 Trautvetter E., Pagel E.B., Skrodzki M. \& Gerlach K. 1990. Changes in the heartbeat of puppies in the first twelve weeks of life. Berliner und Munchener Tierarztliche Wochenschrift. 103(7): 229-232.

20 Van der Berg M., Rijnbeek P.R., Niemeijer M.N., Hofman A., Van Herpen V., Bots M.L., Hillege H., Swenne C.A., Eijgelsheim M., Stricker B. \& Kors J.A. 2018. Normal Values of Corrected Heart-Rate Variability In 10-Second Electrocardiograms for All Ages. Frontiers in Physiology. 9: 1-9.

21 Zupan M., Buskas J., Altimiras J. \& Keeling L.J. 2016. Assessing positive emotional states in dogs using heart rate and heart rate variability. Physiology Behavior. 155: 102-111. 